\title{
Clinical use of linezolid in periprosthetic joint infections - a systematic review
}

\author{
Christoph Theil, Tom Schmidt-Braekling, Georg Gosheger, Jan Schwarze, Ralf Dieckmann, \\ Kristian Nikolaus Schneider, and Burkhard Möllenbeck \\ Department of Orthopedics and Tumor Orthopedics, Muenster University Hospital, \\ Albert-Schweitzer-Campus 1, 48149, Münster, Germany \\ Correspondence: Christoph Theil (christoph.theil@ukmuenster.de)
}

Received: 16 March 2020 - Revised: 18 June 2020 - Accepted: 20 June 2020 - Published: 13 July 2020

\begin{abstract}
Introduction: The most common causative organism in periprosthetic joint infections (PJIs) is Grampositive bacteria that are increasingly drug resistant. In these cases the use of linezolid may be warranted. However, there are conflicting reports regarding its role in antibiotic treatment of PJIs. The aim of this review is to gather and analyze clinical results and treatment details on linezolid in patients with PJIs. Methods: In August 2019, a comprehensive literature search using MEDLINE (Pubmed and Ovid) and Cochrane Library was performed. A total of 504 records were screened, and a total of 16 studies including 372 patients treated with linezolid for a PJI were included in this review based on the PRISMA criteria and after quality analysis using the MINOR score and Newcastle-Ottawa scale, as well as assessing level of evidence. Pooling analysis as well as descriptive analysis was performed. Results: Based on the results from the studies included, infection control was achieved in $80 \%$ (range $30 \%-100 \%$ ) of patients after a mean follow-up period of 25 (range 2-66) months. The mean duration of treatment was $58 \mathrm{~d}$ intravenous and orally at a median dose of $600 \mathrm{mg}$ bis in die (b.i.d.) (range 400-900 b.i.d.). A combination therapy with rifampicin was used in $53 \%$ of patients. MRSA (methicillinresistant Staphylococcus aureus) infections were present in $29 \%$ and resistant CoNS (coagulase-negative Staphylococcus) in $46 \%$. Adverse effects occurred in $33 \%$ of cases, mostly anemia, thrombocytopenia and gastrointestinal complaints leading to treatment discontinuation in $9 \%$. However, great heterogeneity was found with respect to surgical treatment, diagnosis of infection and indication for linezolid. Discussion: Linezolid is an appropriate option for treatment of resistant Gram-positive organisms in PJIs. Most commonly $600 \mathrm{mg}$ b.i.d. is used, and a combination with rifampicin appears feasible although one must consider individual increases in doses in these cases. However, adverse effects are common and there are limited data for long-term use and optimal antibiotic combinations or individual doses.
\end{abstract}

\section{Introduction}

The treatment of periprosthetic joint infection (PJI) includes different surgical approaches involving debridement and prosthesis retention as well as one-stage exchange or twostage exchange, and medical systemic treatment can vary greatly regarding length and substances used (Osmon et al., 2013b; Anemuller et al., 2019; de Beaubien et al., 2019) with successful shorter-term courses (Winkler et al., 2019) described as being contrasted by long-time antibiotic suppression treatment in severe, complicated cases (Siqueira et al., 2015; Wouthuyzen-Bakker et al., 2017; Leijtens et al., 2019). Furthermore, microbiological results are changing with increasing prevalence of resistant strains (Drago et al., 2017; De Vecchi et al., 2018), particularly methicillinresistant (MR) coagulase-negative Staphylococcus (CoNS) now being the main pathogen detected (Lourtet-Hascoet et al., 2018; Hipfl et al., 2019; Tevell et al., 2019). In this context, linezolid is a potential antimicrobial treatment option addressing resistant Staphylococcus (Deroche et al., 2019) as well as reducing the need for long-term inpatient treatment given its excellent oral bioavailability (Kutscha-Lissberg et al., 2003). However, linezolid can have some feared adverse 
effects such as cytopenia, particularly of leukocytes and neuropathy, that might lead to treatment discontinuation (Legout et al., 2010). Furthermore, due to biofilm formation on the infected implant by Staphylococcus, rifampicin as a biofilmactive drug (Zimmerli and Sendi, 2019) could potentially be combined with linezolid in these infections. However, there are concerns regarding adverse effects and drug interactions with this combination (Gomez et al., 2011; Gandelman et al., 2011).

While current consensus statements and widely used treatment guidelines recommend the use of linezolid only for (vancomycin-)resistant Enterococcus or as an alternative treatment for resistant Staphylococcus (Anemuller et al., 2019; de Beaubien et al., 2019; Osmon et al., 2013b), there are several reports that recommend its use as either an empirical treatment (Deroche et al., 2019; Takoudju et al., 2018) or for early oral treatment reducing the need for in-hospital intravenous treatment with good results (Oussedik and Haddad, 2008; Legout et al., 2010; Cobo et al., 2013). Furthermore, in implant-related infections rifampicin and its derivatives needs to be considered as a potential drug for combined treatment considering its anti-biofilm properties in staphylococcal infections (Zimmerli and Sendi, 2019).

A previous review article on linezolid in orthopedic implant infections (Morata et al., 2014a) reported a success rate of around $70 \%$ with adverse effects reported to occur in $34 \%$ of all cases. However, orthopedic implant infections included in this analysis range from very minor infections such as external fixator pin infections to severe prosthetic (re-)infections for which surgical treatment, patient characteristics and common length of treatment as well as success rates are expected to vary greatly (Aboltins et al., 2019; Metsemakers et al., 2018; Moriarty et al., 2016). To our knowledge, there is no review on the use of linezolid for prosthetic joint infections specifically.

The aim of this review is to evaluate the study quality of published articles and to analyze current clinical results as well as treatment details and microbiology findings of PJIs treated with linezolid.

\section{Methods}

A comprehensive literature research of publications until 12 August 2019 using the Pubmed, Ovid Embase and Cochrane Library search was performed. Search terms were "linezolid periprosthetic/prosthetic joint/s infection/s", "linezolid joint/s infection/s", "linezolid joint/s", "linezolid bone" and "linezolid arthroplasty/ies". The search was restricted to studies on humans published between 1950 and August 2019 for papers in English. The review algorithm was based on the Preferred Reporting Items for Systematic Review and Meta-Analyses (PRISMA) criteria (Moher et al., 2009), and search results are presented in a PRISMA conform diagram (Fig. 1).

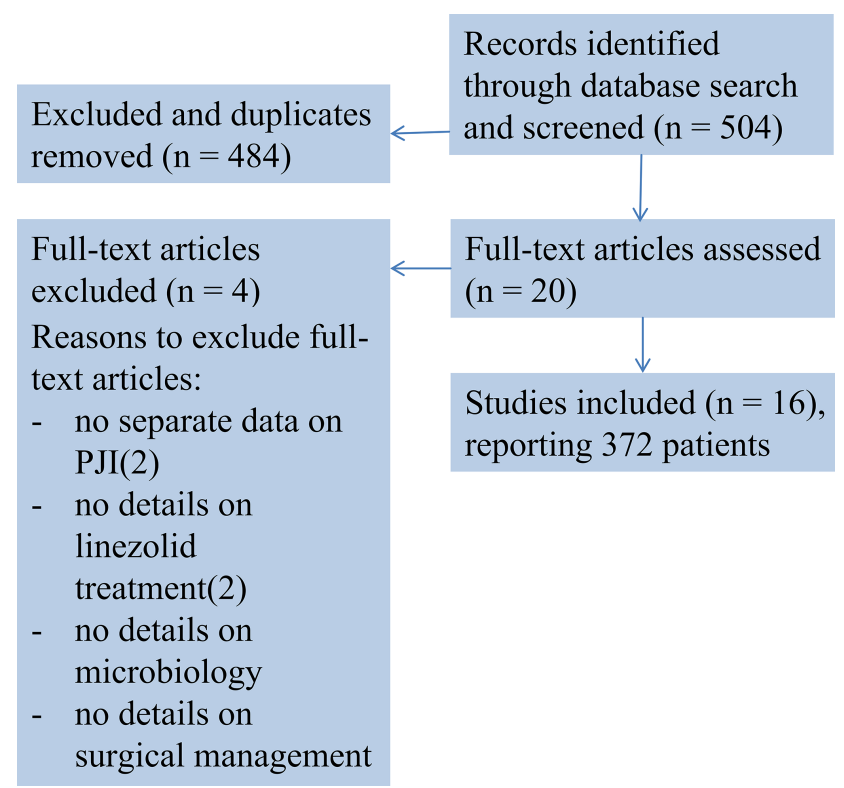

Figure 1. Flowchart showing the studies included.

Titles and abstract were reviewed by two authors (Christoph Theil and Burkhard Möllenbeck). Following exclusion based on title and abstract, a full text was obtained and reviewed by the same investigators. In many cases supplemental materials were obtained and reviewed additionally. The study quality was assessed using the Methodological Index for Non-Randomized Studies (MINORS) checklist (Slim et al., 2003) that allows for the calculation of a quality score (maximum score out of 16 for observational studies and out of 24 for comparative studies) as well as the NewcastleOttawa scale (ranging from 0 to 9 stars). The level of evidence was determined using the Oxford Centre for EvidenceBased Medicine (OCEBM) levels of evidence (OCEBM Levels of Evidence Working Group, 2011).

Inclusion criteria were the use of linezolid in PJIs of hip and knee joint replacements. Case reports, reviews on PJIs and studies with fewer than five patients were excluded. Studies about orthopedic infections that mention linezolid use in PJIs but in which results for treatment of PJIs could not be extracted were excluded (four studies). If data on PJIs were presented among other data on infections in which all patients were treated with linezolid, it was extracted and general statements (e.g., median age or follow-up period) were assumed to be applicable for patients treated with PJIs in these studies. These results are highlighted. Further references were obtained by reviewing general practice guidelines and consensus statements (Osmon et al., 2013a, b; de Beaubien et al., 2019) regarding the use of linezolid in PJIs.

Microbiology details, length of treatment, potential antibiotic combinations as well as adverse effects have been extracted from the studies. The primary outcome measure was infection control as defined by the respective study. Despite 
the heterogeneity of studies and missing values for several variables obtained from the studies presented in terms of surgical management, definition of infection as well as administration and length of antibiotic treatment, we chose to perform a simple pooling of the aggregate results for the outcome measures when possible and a descriptive analysis. Descriptive statistics were used to analyze distribution of data. Weighed (based on the number of cases per study included in the analysis of different outcome measures) means and ranges were calculated for parametric data and medians and interquartile ranges (IQRs) for nonparametric data. Due to the heterogeneity encountered no further statistical analysis or meta-analysis was performed.

Statistical analysis was performed using Microsoft Excel 2016 (Microsoft Corporation, Redmond, Washington, USA) and SPSS Statistics for Windows Version 25 (IBM Corporation, Armonk, New York, USA).

\section{Results}

\subsection{Study types and quality}

We were able to identify a total of 16 studies that met the inclusion criteria. The studies included in this article report the outcome of a total of 372 patients treated with linezolid for a PJI.

There were no randomized controlled studies, and all studies were of observational nature. Nine studies were retrospective single or multicenter studies while seven studies were prospective single or multicenter studies reporting results on a mean of 16 patients (range 8-53) with a mean age of 64 years (range 54-76) after a mean follow-up period of 25 months (range 2-66) (Table 1).

\subsection{Infection control}

The infection was initially controlled in $80 \%(229 / 285)$ of cases included for that measure with three studies only reporting a range (Harwood et al., 2006; Rao and Hamilton, 2007; Soriano et al., 2007) (Table 1) because of heterogeneous patient cohorts. Additionally, five studies reported a separate median reinfection rate during the respective followup period (Bassetti et al., 2005; Cobo et al., 2013; Eriksson et al., 2019; Morata et al., 2014a; Oussedik and Haddad, 2008) with a mean of $22 \%$ (26/120 patients). Based on the available information and different definitions, $48 \%$ (range $0-100 \%$ ) of all PJIs can be considered early infections $(119 / 244)$ and $52 \%$ of all PJIs were infected TKA (total knee arthroplasty) (112/217).

\subsection{Indications, treatment details and microbiological details}

The indications for the use of linezolid varied between the studies. Two studies included all patients with Gram- positive PJIs (Bassetti et al., 2005; Cobo et al., 2013). Six studies reported resistant bacteria as an indication (Eriksson et al., 2019; Morata et al., 2014a; Nguyen et al., 2009; Papadopoulos et al., 2009; Rao and Hamilton, 2007; Razonable et al., 2004). Failure or intolerance of previous treatment was noted in nine studies (Eriksson et al., 2019; Harwood et al., 2006; Gomez et al., 2011; Legout et al., 2010; Lu et al., 2010; Rao and Hamilton, 2007; Razonable et al., 2004; Soriano et al., 2007; Papadopoulos et al., 2009) or oral application following intravenous treatment in two studies (Joel et al., 2014; Oussedik and Haddad, 2008). The mean length of treatment in the included studies (combined intravenously and orally) was $58 \mathrm{~d}(25-125 \mathrm{~d})$. Prior to the use of linezolid, five studies reported a different intravenous treatment (Eriksson et al., 2019; Gomez et al., 2011; Harwood et al., 2006; Joel et al., 2014; Oussedik and Haddad, 2008) or antibiotic combined treatment for polymicrobial infections in up to $35 \%$ of patients (Legout et al., 2010). With regard to the role of rifampicin-linezolid combinations, eight studies report no parallel use of rifampicin and linezolid, while on the other hand eight studies (Legout et al., 2010; Joel et al., 2014; Eriksson et al., 2019; Soriano et al., 2007; Tornero et al., 2016; Morata et al., 2014a; Gomez et al., 2011; Nguyen et al., 2009) generally use this combination when sensitive organisms and Staphylococcal infection were present in a mean of $53 \%$ of cases $(131 / 246$ patients, range 3\%-100\%) in the respective studies.

As expected the most commonly isolated microorganism was Staphylococcus. Six studies (Harwood et al., 2006; Lu et al., 2010; Bassetti et al., 2005; Razonable et al., 2004; Legout et al., 2010; Nguyen et al., 2009) reported methicillin-resistant Staphylococcus aureus (MRSA) as the main pathogen in a mean of $29 \%$ (range $0 \%-85 \%$ ) of cases, while otherwise coagulase-negative Staphylococcus was the most common organism with a mean of $46 \%$ (range 15100) of patients. Five studies also used linezolid in culturenegative infections (Gomez et al., 2011; Papadopoulos et al., 2009; Harwood et al., 2006; Soriano et al., 2007; Lu et al., 2010), mostly as a second-line treatment, with a median percentage of culture-negative infections in these studies of $14 \%$ (IQR $11 \%-25 \%$ ). On the other hand polymicrobial infection was reported in 11 studies (Joel et al., 2014; Tornero et al., 2016; Morata et al., 2014a; Razonable et al., 2004; Legout et al., 2010; Soriano et al., 2007; Cobo et al., 2013; Oussedik and Haddad, 2008; Harwood et al., 2006; Rao and Hamilton, 2007; Nguyen et al., 2009) with a median percentage of $8 \%$ of patients included (IQR $2 \%-37 \%$ ).

Microbiology findings, treatment details and antibiotic duration are summarized in Table 2.

Adverse effects were reported in $94 \%(15 / 16)$ of studies included. The mean frequency of adverse events was $33 \%$ (range $7 \%-76 \%$ ). The most common complications were hematological alterations; $75 \%(12 / 16)$ of studies included report a percentage of patients who discontinued treatment at a mean rate of $9 \%$ (range $0 \%-44 \%$ ) (Table 3 ). 
Table 1. Study quality and methodological assessment.

\begin{tabular}{|c|c|c|c|c|c|c|}
\hline & Design & $\begin{array}{l}\text { No. of } \\
\text { patients } \\
\text { included }\end{array}$ & $\begin{array}{l}\text { Follow-up } \\
\text { period in } \\
\text { months }\end{array}$ & $\begin{array}{l}\text { MINORS } \\
\text { score (out } \\
\text { of } 16 \text { if not } \\
\text { otherwise } \\
\text { indicated) }\end{array}$ & $\begin{array}{l}\text { Newcastle- } \\
\text { Ottawa } \\
\text { score }\end{array}$ & $\begin{array}{l}\text { Level of } \\
\text { evidence } \\
\text { (Oxford) }\end{array}$ \\
\hline Cobo et al. (2013) & $\begin{array}{l}\text { prospective, } \\
\text { multicenter }\end{array}$ & 25 & 14 & 13 & 5 & 3 \\
\hline Bassetti et al. (2005) & $\begin{array}{l}\text { retrospective, } \\
\text { single center }\end{array}$ & 20 & 12 & 11 & 5 & 4 \\
\hline Gomez et al. (2011) & $\begin{array}{l}\text { prospective, } \\
\text { single center }\end{array}$ & 49 & 24 & 14 & 6 & 3 \\
\hline Harwood et al. (2006) & $\begin{array}{l}\text { prospective, } \\
\text { single center }\end{array}$ & 11 & 13 & 8 & 5 & 4 \\
\hline Joel et al. (2014) & $\begin{array}{l}\text { retrospective, } \\
\text { single center }\end{array}$ & 10 & 34 & 12 & 5 & 4 \\
\hline Legout et al. (2010) & $\begin{array}{l}\text { retrospective, } \\
\text { multicenter }\end{array}$ & 39 & 16 & 10 & 7 & 3 \\
\hline Lu et al. (2010) & $\begin{array}{l}\text { prospective, } \\
\text { multicenter }\end{array}$ & 17 & 6 & 8 & 5 & 4 \\
\hline Morata et al. (2014a) & $\begin{array}{l}\text { retrospective, } \\
\text { multicenter }\end{array}$ & 38 & 25 & $18 / 24$ & 7 & 3 \\
\hline Nguyen et al. (2009) & $\begin{array}{l}\text { retrospective, } \\
\text { multicenter }\end{array}$ & 11 & 24 & $17 / 24$ & 7 & 3 \\
\hline $\begin{array}{l}\text { Oussedik and Haddad } \\
(2008)\end{array}$ & $\begin{array}{l}\text { retrospective, } \\
\text { single center }\end{array}$ & 14 & 33 & 12 & 6 & 4 \\
\hline Papadopoulos et al. (2009) & $\begin{array}{l}\text { prospective, } \\
\text { case-control } \\
\text { study }\end{array}$ & 8 & 2 & 12 & 7 & 3 \\
\hline Rao and Hamilton (2007) & $\begin{array}{l}\text { prospective, } \\
\text { single center }\end{array}$ & 23 & 19 & 11 & 5 & 3 \\
\hline Razonable et al. (2004) & $\begin{array}{l}\text { retrospective, } \\
\text { single center }\end{array}$ & 8 & 7 & 10 & 5 & 4 \\
\hline Soriano et al. (2007) & $\begin{array}{l}\text { prospective, } \\
\text { multicenter }\end{array}$ & 53 & $\begin{array}{l}\text { Min. } \\
12-47^{*}\end{array}$ & 14 & 7 & 3 \\
\hline Tornero et al. (2016) & $\begin{array}{l}\text { retrospective, } \\
\text { single center }\end{array}$ & 17 & $66^{*}$ & $18 / 24$ & 7 & 3 \\
\hline Eriksson et al. (2019) & $\begin{array}{l}\text { retrospective, } \\
\text { single center }\end{array}$ & 28 & 51.6 & 12 & 6 & 4 \\
\hline
\end{tabular}

* No PJI-specific results.

\section{Discussion}

Linezolid offers the advantage of very good, oral bioavailability (Thompson et al., 2017) and its broad spectrum against Gram-positive bacteria facing current resistance patterns (Deroche et al., 2019; Lourtet-Hascoet et al., 2018) of organisms encountered in the treatment of PJI. Based on the reports available and included in this review a remission of the infection can be expected in around $80 \%$ of cases. However, there are several issues that surgeons and infectious disease specialists need to consider in this environment.

While this review reports on a large number of patients treated with linezolid and the results presented can help in planning antimicrobial treatment as it provides an overview about adverse effects, expected rates of infection control and potential antibiotic combinations, there are limitations to this 
Table 2. Systemic and local treatment details, microbiological findings.

\begin{tabular}{|c|c|c|c|c|c|c|c|}
\hline Study & Type of infection & Indication linezolid & $\begin{array}{l}\text { Duration } \\
\text { and } \\
\text { treatment } \\
\text { details }\end{array}$ & Linezolid dose & Combination with other antibiotics & $\begin{array}{l}\text { Surgical } \\
\text { management }\end{array}$ & Microbiology \\
\hline $\begin{array}{l}\text { Cobo et al. } \\
\text { (2013) }\end{array}$ & chronic & $\begin{array}{l}\text { all Gram-positive } \\
\text { infections }\end{array}$ & $\begin{array}{l}42 \mathrm{~d} \text { (IV or } \\
\text { oral) }\end{array}$ & 600 b.i.d. & $\begin{array}{l}\text { permitted antibiotic combinations for } \\
\text { polymicrobial infection, long-term clin- } \\
\text { damycin in one case }\end{array}$ & two-stage & $\begin{array}{l}81 \% \text { Staphylococcus, } 19 \% \\
\text { Streptococcus and others, no } \\
\text { MRSA, 33\% MRSE } \\
\text { PM } 8 \% \\
\text { CN: } 0 \%\end{array}$ \\
\hline $\begin{array}{l}\text { Bassetti et } \\
\text { al. (2005) }\end{array}$ & $\begin{array}{l}45 \% \text { acute } \\
55 \% \text { chronic }\end{array}$ & $\begin{array}{l}\text { all Gram-positive } \\
\text { infections }\end{array}$ & $\begin{array}{l}50.4 \mathrm{~d} \text { (IV } \\
\text { and oral) }\end{array}$ & not reported & $\begin{array}{l}75 \% \text { patients previous treatment, } 55 \% \\
\text { ciprofloxacin-rifampicin combination, } \\
20 \% \text { glycopeptide }\end{array}$ & $\begin{array}{l}\text { DAIR or single } \\
\text { stage }\end{array}$ & $\begin{array}{l}70 \% \text { MRSA, } 25 \% \text { MRSE, } 5 \% \\
\text { Enterococcus } \\
\text { PM } 0 \% \\
\text { CN: } 0 \%\end{array}$ \\
\hline $\begin{array}{l}\text { Gomez et } \\
\text { al. }(2011)\end{array}$ & $\begin{array}{l}63 \% \text { early } \\
(<30 \mathrm{~d}) 37 \% \text { late } \\
(>30 \mathrm{~d})\end{array}$ & failed prior treatment & $80.2 \mathrm{~d}$ oral & 600 b.i.d. & $\begin{array}{l}100 \% \text { combination with rifampicin, } \\
\text { otherwise ciprofloxacin, teicoplanin, } \\
\text { cotrimoxazole }\end{array}$ & $\begin{array}{l}77.8 \% \text { DAIR, } \\
22.8 \% \text { non- } \\
\text { operative }\end{array}$ & $\begin{array}{l}45 \% \text { MRSE, } 12 \% \text { MRSA } \\
\text { PM } 0 \% \\
\text { CN: } 35 \%\end{array}$ \\
\hline $\begin{array}{l}\text { Harwood } \\
\text { et } \\
\text { al. (2006) }\end{array}$ & $\mathrm{n} / \mathrm{a}$ & $\begin{array}{l}\text { intolerance of gly- } \\
\text { copeptide, failed } \\
\text { prior treatment, oral } \\
\text { continuation }\end{array}$ & $39 \mathrm{~d}$ oral $^{*}$ & not reported & $\begin{array}{l}\text { previous or combination: } \\
\text { flucloxacillin, cephalosporin, } \\
\text { vancomycin, rifampicin }\end{array}$ & $\begin{array}{l}18 \% \text { non- } \\
\text { operative, } 27 \% \\
\text { DAIR, } 54 \% \\
\text { two-stage }\end{array}$ & $\begin{array}{l}85 \% \text { MRSA, } 15 \% \text { CoNS } \\
\text { PM } 6 \% \\
\text { CN: } 2 \%\end{array}$ \\
\hline $\begin{array}{l}\text { Joel et al. } \\
\text { (2014) }\end{array}$ & $\mathrm{n} / \mathrm{a}$ & $\begin{array}{l}\text { oral continuation of } \\
\text { therapy }\end{array}$ & $30 \mathrm{~d}$ oral & not reported & $\begin{array}{l}\text { previous treatment: } \\
\text { flucloxacillin, cephalosporin, } \\
\text { vancomycin, rifampicin }\end{array}$ & $\mathrm{n} / \mathrm{a}$ & $\begin{array}{l}70 \% \text { CoNS, } 10 \% \text { MRSA, } 10 \% \\
\text { Enterococcus, } 10 \% \text { Staphylo- } \\
\text { coccus } \\
\text { aureus } \\
\text { PM } 0 \% \\
\text { CN: } 11.3 \%\end{array}$ \\
\hline $\begin{array}{l}\text { Legout et } \\
\text { al. (2010) }\end{array}$ & $\mathrm{n} / \mathrm{a}$ & $\begin{array}{l}\text { contraindications for } \\
\text { other, vancomycin } \\
\text { intolerance }\end{array}$ & $\begin{array}{l}101.5 \mathrm{~d} \\
\text { oral }^{*}\end{array}$ & 600 b.i.d. & $\begin{array}{l}\text { combination with fluoroquinolones, } \\
\text { beta-lactams, others }\end{array}$ & $\begin{array}{l}\text { DAIR, one } \\
\text { stage, two- } \\
\text { stage }\end{array}$ & $\begin{array}{l}36 \% \text { MRSA, } 21 \% \text { MRSE, } 6 \% \\
\text { Enterococcus } * \\
\text { PM } 38 \% \\
\text { CN: } 0 \%\end{array}$ \\
\hline $\begin{array}{l}\text { Lu et al. } \\
\text { (2010) }\end{array}$ & $\mathrm{n} / \mathrm{a}$ & $\begin{array}{l}\text { failure or intolerance of } \\
\text { other treatment }\end{array}$ & $\begin{array}{l}25 \mathrm{~d} \mathrm{IV} \text { and } \\
\text { oral* }^{*}\end{array}$ & 600 b.i.d. & $\begin{array}{l}30 \text { with vancomycin and } 25 \text { with te- } \\
\text { icoplanin, and } 5 \text { cases received fusidic } \\
\text { acid, } 2 \text { gentamicin, } 2 \text { ciprofloxacin, } 1 \\
\text { trimethoprim/sulfamethoxazole, } 1 \text { cefa- } \\
\text { zolin, } 1 \text { rifampicin, and } 1 \text { oxacillin }\end{array}$ & $\mathrm{n} / \mathrm{a}$ & $\begin{array}{l}79 \% \text { MRSA, } 9.4 \% \text { MSSA }^{*} \\
\text { PM } 0 \% \\
\text { CN: } 11.3 \%\end{array}$ \\
\hline $\begin{array}{l}\text { Morata et } \\
\text { al. (2014a) }\end{array}$ & $\begin{array}{l}90 \% \text { acute } \\
(<4 \text { weeks }), 10 \% \\
\text { late acute }\end{array}$ & $\mathrm{n} / \mathrm{a}$ & $\begin{array}{l}44.5 \mathrm{~d} \text { IV } \\
\text { and oral }\end{array}$ & $\begin{array}{l}600 \text { b.i.d., up to } \\
900 \text { b.i.d. with } \\
\text { rifampicin }\end{array}$ & $\begin{array}{l}\text { ciprofloxacin, beta-lactam for polymi- } \\
\text { crobial infection }\end{array}$ & DAIR & $\begin{array}{l}61 \% \text { CoNS, } 13 \% \text { MRSA, } 7 \% \\
\text { Enterococcus } \\
\text { PM } 38 \% \\
\text { CN: } 0 \%\end{array}$ \\
\hline $\begin{array}{l}\text { Nguyen et } \\
\text { al. (2009) }\end{array}$ & $\begin{array}{l}\text { chronic }>30 \mathrm{~d} \text { of infec- } \\
\text { tion, }>2 \text { months post- } \\
\text { operatively }\end{array}$ & $\begin{array}{l}\text { Gram-positive coccal } \\
\text { infection }\end{array}$ & $\begin{array}{l}124.6 \mathrm{~d} \text { IV } \\
\text { and oral* }\end{array}$ & 600 b.i.d. & glycopeptide, cephalosporin & $\begin{array}{l}27 \% \text { one-stage, } \\
27 \% \text { two-stage, } \\
36 \% \text { DAIR, } \\
9 \% \text { resection } \\
\text { arthroplasty }\end{array}$ & $\begin{array}{l}34.4 \% \text { MRSA, } 28.1 \% \text { CoNS, } \\
\text { 15.6. } \% \text { Enterococcus } * \\
\text { PM } 3.1 \% \% \\
\text { CN: } 0 \%\end{array}$ \\
\hline $\begin{array}{l}\text { Oussedik } \\
\text { and } \\
\text { Haddad } \\
(2008)\end{array}$ & $\begin{array}{l}71 \% \text { chronic, } 29 \% \\
\text { early or intermediate }\end{array}$ & oral treatment & $37.1 \mathrm{~d}$ oral & 600 b.i.d. & Teicoplanin & $\begin{array}{l}85 \% \text { two-stage, } \\
7 \% \text { DAIR, } 7 \% \\
\text { one-stage }\end{array}$ & $\begin{array}{l}57 \% \text { CoNS, } 29 \% \text { MRSA, } 15 \% \\
\text { MSSA } \\
\text { PM } 7 \% \\
\text { CN: } 0 \%\end{array}$ \\
\hline $\begin{array}{l}\text { Papadopoulos } \\
\text { et al. (2009) }\end{array}$ & $\mathrm{n} / \mathrm{a}$ & $\begin{array}{l}\text { resistant bacteria, intol- } \\
\text { erance of glycopeptide }\end{array}$ & $\begin{array}{l}42 \mathrm{~d} \text { i.v and } \\
\text { oral }\end{array}$ & 600 b.i.d. & none & $\begin{array}{l}62.5 \% \text { non- } \\
\text { operative, } \\
37.5 \% \text { staged } \\
\text { revisions }\end{array}$ & $\begin{array}{l}50 \% \text { MRSE, } 25 \% \text { MRSA } \\
\text { PM } 0 \% \\
\text { CN: } 25 \%\end{array}$ \\
\hline $\begin{array}{l}\text { Rao and } \\
\text { Hamilton } \\
\text { (2007) }\end{array}$ & $\mathrm{n} / \mathrm{a}$ & $\begin{array}{l}\text { intolerance, failure, re- } \\
\text { sistance to vancomycin }\end{array}$ & $\begin{array}{l}42 \mathrm{~d} \text { IV and } \\
\text { oral }\end{array}$ & 600 b.i.d. & $\begin{array}{l}\text { possible for Gram-negative or fungal } \\
\text { infection, suppression therapy in se- } \\
\text { lected cases using cephalexin, minocy- } \\
\text { cline, trimethoprim, fluoroquinolones }\end{array}$ & $\begin{array}{l}61 \% \text { DAIR, } \\
39 \% \text { staged } \\
\text { revision }\end{array}$ & $\begin{array}{l}39 \% \text { MRCoNS, } 22 \% \text { MRSA, } 2 \\
\text { Enterococcus } \\
\text { PM } 4 \% \\
\text { CN: } 0 \%\end{array}$ \\
\hline $\begin{array}{l}\text { Razonable } \\
\text { et al. } \\
(2004)\end{array}$ & $\begin{array}{l}63 \% \text { chronic, } 37 \% \\
\text { acute }\end{array}$ & $\begin{array}{l}\text { vancomycin resis- } \\
\text { tance, intolerance of } \\
\text { vancomycin, failure of } \\
\text { vancomycin }\end{array}$ & $49 \mathrm{~d}$ oral & $\begin{array}{l}600 \text { b.i.d., low- } \\
\text { ered to } \\
400 \text { b.i.d. in } \\
\text { two patients }\end{array}$ & $\begin{array}{l}\text { fluoroquinolones, cephalosporin, beta- } \\
\text { lactam, fluconazole depending on } \\
\text { microbiology }\end{array}$ & $\begin{array}{l}75 \% \text { resection } \\
\text { arthroplasty, } \\
25 \% \text { DAIR }\end{array}$ & $\begin{array}{l}50 \% \text { MRSA, } 50 \% \text { CoNS, } 25 \% \\
\text { VRE } \\
\text { PM } 37 \% \\
\text { CN: } 0 \%\end{array}$ \\
\hline $\begin{array}{l}\text { Soriano et } \\
\text { al. (2007) }\end{array}$ & $\begin{array}{l}28 \% \text { acute, } 72 \% \\
\text { chronic }\end{array}$ & $\begin{array}{l}\text { oral continuation of } \\
\text { therapy, failure or } \\
\text { intolerance of previous } \\
\text { treatment }\end{array}$ & $56 \mathrm{~d}$ oral $^{*}$ & 600 b.i.d. & in polymicrobial infections & $\begin{array}{l}63 \% \text { implant } \\
\text { retention*, } \\
37 \% \text { staged } \\
\text { revision }\end{array}$ & $\begin{array}{l}\text { n/a } \\
\text { PM } 16 \% \\
\text { CN: } 14 \%\end{array}$ \\
\hline $\begin{array}{l}\text { Tornero et } \\
\text { al. (2016) }\end{array}$ & acute (within $90 \mathrm{~d}$ ) & $\mathrm{n} / \mathrm{a}$ & $\begin{array}{l}76 \mathrm{~d} \mathrm{IV} \text { and } \\
\text { oral* }^{*}\end{array}$ & 600 b.i.d. & $\begin{array}{l}\text { vancomycin and ceftazidime, } \\
\text { rifampicin in } 8 \text { out of } 15 \text { cases }\end{array}$ & DAIR & $\begin{array}{l}\text { CoNS } 48 \% \text {, Staphylococcus } \\
\text { aureus } \\
37 \% \text {, Enterococcus } 13 \% * \\
\text { PM } 39 \% \\
\text { CN: n/a }\end{array}$ \\
\hline $\begin{array}{l}\text { Eriksson et } \\
\text { al. (2019) }\end{array}$ & $\begin{array}{l}58 \% \text { early } \\
\text { ( }<3 \text { months), } 39 \% \\
\text { delayed ( } 3-24 \text { months), } \\
3 \% \text { late ( }>24 \text { months) }\end{array}$ & $\begin{array}{l}\text { intolerance of other } \\
\text { treatment }(5 / 28) \text {, } \\
\text { resistant CoNS }\end{array}$ & $29.4 \mathrm{~d}$ oral & 600 b.i.d. & $\begin{array}{l}\text { vancomycin } 22 / 28 \text {, teicoplanin } 3 / 28 \text {, } \\
\text { cloxacillin } 2 / 28 \text {, daptomycin } 1 / 28\end{array}$ & $\begin{array}{l}54 \% \text { 2-stage } \\
\text { exchange, } 46 \% \\
\text { DAIR }\end{array}$ & $\begin{array}{l}\text { CoNS }(16 / 28) \\
\text { PM n/a } \\
\text { CN: } 0 \%\end{array}$ \\
\hline
\end{tabular}


Table 3. Adverse effects reported.

\begin{tabular}{|c|c|c|c|}
\hline Study & $\begin{array}{l}\% \text { of } \\
\text { adverse } \\
\text { effects }\end{array}$ & $\begin{array}{l}\% \text { of } \\
\text { discontinuation }\end{array}$ & Types of adverse effects \\
\hline Cobo et al. (2013) & $76 \%$ & $13 \%$ & $\begin{array}{l}76 \% \text { thrombocytopenia } \\
40 \% \text { nausea } \\
36 \% \text { anemia }\end{array}$ \\
\hline Bassetti et al. (2005) & $15 \%$ & $0 \%$ & $15 \%$ gastrointestinal symptoms none hematological \\
\hline Gomez et al. (2011) & $36.6 \%$ & $0 \%$ & $\begin{array}{l}12 \% \text { candidiasis and gastrointestinal discomfort } \\
6 \% \text { thrombocytopenia } \\
6 \% \text { anemia }\end{array}$ \\
\hline Harwood et al. (2006) & $44 \%$ & $19 \%^{2}$ & $\begin{array}{l}15 \% \text { anemia } \\
15 \% \text { nausea vomiting } \\
11 \% \text { diarrhea }\end{array}$ \\
\hline Joel et al. (2014) & $10 \%$ & $10 \%$ & $10 \%$ thrombocytopenia \\
\hline Legout et al. (2010) & $48 \%$ & $15 \% *$ & $\begin{array}{l}48 \% \text { thrombocytopenia } \\
29 \% \text { anemia } \\
9 \% \text { neuropathy }\end{array}$ \\
\hline Lu et al. (2010) & $25 \%$ & $\begin{array}{l}11.3 \% \\
\text { discontinued }\end{array}$ & $\begin{array}{l}25 \% \text { thrombocytopenia } \\
18 \% \text { anemia } \\
6 \% \text { leukopenia } \\
\text { no neuropathy }\end{array}$ \\
\hline Morata et al. (2014a) & $38 \%$ & $0 \%$ & $\begin{array}{l}26 \% \text { gastrointestinal } \\
13 \% \text { hematological } \\
5 \% \text { neurotoxicity }\end{array}$ \\
\hline Nguyen et al. (2009) & $42.9 \%$ & $14.3 \% *$ & $\begin{array}{l}14 \% \text { anemia } \\
14 \% \text { gastrointestinal } \\
7 \% \text { hepatic enzyme elevation }\end{array}$ \\
\hline $\begin{array}{l}\text { Oussedik and Haddad } \\
\text { (2008) }\end{array}$ & $7 \%$ & $0 \%$ & $7 \%$ pancytopenia \\
\hline Papadopoulos et al. (2009) & $33 \%$ & $44 \% *$ & $\begin{array}{l}33 \% \text { anemia } \\
9 \% \text { thrombocytopenia } \\
6 \% \text { GIT symptoms } \\
\text { no neuropathy }\end{array}$ \\
\hline Rao and Hamilton (2007) & $22 \%$ & $13 \%$ & $\begin{array}{l}17 \% \text { thrombocytopenia } \\
9 \% \text { GIT symptoms } \\
9 \% \text { anemia }\end{array}$ \\
\hline Razonable et al. (2004) & $50 \%$ & $0 \%$ & $\begin{array}{l}50 \% \text { leukopenia } \\
25 \% \text { thrombocytopenia } \\
13 \% \text { neuropathy }\end{array}$ \\
\hline Soriano et al. (2007) & $13 \%$ & $0 \%$ & $\begin{array}{l}12.9 \% \text { GI symptoms } \\
4.7 \% \text { thrombocytopenia } \\
5.8 \% \text { anemia } \\
\text { no neuropathy* }\end{array}$ \\
\hline Tornero et al. (2016) & $\mathrm{n} / \mathrm{a}$ & $\mathrm{n} / \mathrm{a}$ & $\mathrm{n} / \mathrm{a}$ \\
\hline Eriksson et al. (2019) & $39 \%$ & $14 \%$ & $\begin{array}{l}21 \% \text { anemia } \\
7 \% \text { thrombocytopenia } \\
4 \% \text { leukopenia }\end{array}$ \\
\hline
\end{tabular}

PM - polymicrobial; CN - culture negative; CoNS - coagulase-negative Staphylococcus; DAIR - debridement, antibiotics, irrigation, retention; GIT gastrointestinal; $\mathrm{n} / \mathrm{a}-$ not available. ${ }^{*}$ No PJI-specific results. 
study. As with most reviews, we relied on published data and had several missing values for some variables due to this. We still chose to pool some of the data provided despite heterogeneity of the different studies regarding the indication, definition of infection and surgical treatment as this can be assumed to be the case in everyday practice. As the most common treatment approach for which data could be extracted was implant retention for early or acute infection, we chose to present the results of these patients separately and pool the results regarding outcome. However, even for "early" infections, the definition varies across studies from patients having symptoms of infection for only $2-3$ weeks in some studies and up to 3 months following primary surgery in others (Tornero et al., 2016). A further limitation that needs to be considered when interpreting the results of the studies included in this review is that one main indication for the use of linezolid reported was failure of the previous treatment. Given that repeat staged prosthetic revisions and further septic surgeries following treatment of a PJI are known to lead to much worse results (Kheir et al., 2017; Khan et al., 2019) with regard to remission of an infection, the results discussed here might be low-end estimates of the potential success rate when using linezolid in PJI patients. However, this might reflect current recommendations regarding the use of linezolid as a second-line reserve therapy (Osmon et al., 2013b; Aboltins et al., 2019; Sendi and Zimmerli, 2012). Furthermore, antibiotic susceptibilities might change (Tevell et al., 2019) even in the short-term between treatment stages of a two-stage exchange (George et al., 2018) potentially necessitating the use of linezolid due increased resistance in staged interventions if further revision is required.

In a previous review article Morata et al. (2014b) differentiated treatment success using linezolid based on the surgical approach, which is certainly an important factor that needs to be taken into account when comparing the results of a specific drug presented by different authors. Soriano et al. (2007) for instance reported remission in $38.7 \%$ of chronic PJIs treated with implant retention versus $83 \%-100 \%$ remission using a staged implant exchange while treating both groups with linezolid. Future studies should focus on reporting results using current uniform definitions for infection (Parvizi et al., 2018; Signore et al., 2019) as well as standardized treatment algorithms including details on surgical and medical treatment (Sendi and Zimmerli, 2012).

The most common organism isolated when linezolid was used is resistant specimens of Staphylococcus aureus and coagulase-negative Staphylococcus. Considering the general consensus that rifampicin and its derivatives play a vital role in combating the biofilm on implants (Zimmerli and Sendi, 2019) when treating PJIs, linezolid needs to be evaluated with regard to linezolid-rifampicin-based combination treatment. While an in vitro study (Thompson et al., 2017) suggested the use of such an oral-only treatment regiment, there is conflicting evidence regarding clinical data on rifampicin containing regimens. While Legout et al. (2010) found a lower incidence of anemia when combining rifampicin and linezolid with no difference in remissions, there are two clinical studies (Morata et al., 2014a; Tornero et al., 2016) from one institution that found that a combination treatment was associated with a higher rate of relapse and ultimately treatment failure compared to a linezolid monotherapy or other quinolone-based combinations. This effect is potentially due to the interaction in the cytochrome P-based metabolism of linezolid that is increased when rifampicin is added (Gandelman et al., 2011). The available concentration of linezolid might therefore drop beneath the respective minimal inhibitory concentration (MIC) needed to eliminate the bacteria (Tornero et al., 2016). Therefore, while a combination of linezolid and rifampicin might be desirable in staphylococcal PJI, it has potential adverse effects on the desired control of infection. For future studies, different dosing regimens of linezolid could be evaluated given that some patients appear to be at subtherapeutic levels with the standard dose (Pea et al., 2010), and there currently is no study on the pharmacokinetics of linezolid in patients treated for PJI. In this context, rifampicin could be reevaluated as a useful partner for linezolid.

The optimal antibiotic treatment length in PJI is currently unknown (Aboltins et al., 2019). However, long-term or life-long treatment algorithms were recommended by some guidelines and authors (Leijtens et al., 2019; Osmon et al., 2013b; Calabro et al., 2019; Aboltins et al., 2019), and duration of treatment might play a vital role (Tattevin et al., 2006) - raising the question of whether linezolid, given its adverse effects, is a potential option for long-term treatment or even suppression. In a study on chronic infections not limited to orthopedic infections, Vazquez et al. (2016) concluded that with monitoring of adverse effects, a long-term treatment of greater than 6 weeks can be safely performed. Additionally, therapeutic drug monitoring has proven effective in optimizing dosing regiments in non-orthopedic infection (Pea et al., 2012) and should be implemented in the treatment of PJIs as well, particularly considering that some patients might benefit from dose escalation or de-escalation to ensure adequate MICs and potentially reduce the high percentage of therapy discontinuation in PJI patients reported by some authors (Legout et al., 2010; Harwood et al., 2006).

Other options in the treatment of Gram-positive infections include aminoglycosides, which have the additional advantage of providing excellent elution characteristics from bone cement and can be used as a local treatment, particularly combined with glycopeptides (Badha et al., 2019). However, as a relevant amount of these drugs might be absorbed, systemic complications must be monitored for at least 8 weeks especially if systemic treatment is performed as well (Edelstein et al., 2018). Furthermore, patients with repeat revision or reinfection might be at high risk of developing resistant strains when aminoglycosides were used in a previous surgery (Corona et al., 2014). 
In conclusion, despite its long-term use, potential in combating increasingly resistant Staphylococcus and generally successful results, there are still several questions that need to be answered regarding the role of linezolid, its optimal treatment modality and potential antibiotic combination. Longer courses of treatment require close surveillance, and patients at risk of non-optimal dosage should undergo drug monitoring.

Data availability. All underlying data are in the text and tables.

Author contributions. CT reviewed the literature, performed conception and design tasks, acquired data, analyzed and interpreted data, did the statistical analysis, and drafted the manuscript; TSB analyzed and interpreted data, drafted the manuscript and critically revised the manuscript; GG performed conception and design tasks and critically revised the manuscript; JS performed conception and design tasks and critically revised the manuscript; RD performed conception and design tasks and critically revised the manuscript; KNS performed conception and design tasks and critically revised the manuscript; BM reviewed the literature, performed conception and design tasks, acquired data, analyzed and interpreted data, drafted the manuscript, and critically revised the manuscript

Competing interests. The authors declare that they have no conflict of interest.

Acknowledgements. We acknowledge and thank the openaccess publication fund of Westphalian Wilhelms University of Münster (WWU Münster) for paying the article processing charge.

Financial support. This research has been supported by Westphalian Wilhelms University of Münster (WWU Münster).

Review statement. This paper was edited by Parham Sendi.

\section{References}

Aboltins, C. A., Berdal, J. E., Casas, F., Corona, P. S., Cuellar, D., Ferrari, M. C., Hendershot, E., Huang, W., Kuo, F. C., Malkani, A., Reyes, F., Rudelli, S., Safir, O., Seyler, T., Tan, T. L., Townsend, R., Tuncay, I., Turner, D., Winkler, H., WouthuyzenBakker, M., Yates, A. J., and Zahar, A.: Hip and Knee Section, Prevention, Antimicrobials (Systemic): Proceedings of International Consensus on Orthopedic Infections, J. Arthroplasty, 34, S279-S288, https://doi.org/10.1016/j.arth.2018.09.012, 2019.

Anemuller, R., Belden, K., Brause, B., Citak, M., Del Pozo, J. L., Frommelt, L., Gehrke, T., Hewlett, A., Higuera, C. A., Hughes, H., Kheir, M., Kim, K. I., Konan, S., Lausmann, C., Marculescu, C., Morata, L., Ramirez, I., Rossmann, M., Sili- bovsky, R., Soriano, A., Suh, G. A., Vogely, C., Volpin, A., Yombi, J., Zahar, A., and Zimmerli, W.: Hip and Knee Section, Treatment, Antimicrobials: Proceedings of International Consensus on Orthopedic Infections, J. Arthroplasty, 34, S463-S475, https://doi.org/10.1016/j.arth.2018.09.032, 2019.

Badha, V., Moore, R., Heffernan, J., Castaneda, P., McLaren, A., and Overstreet, D.: Determination of Tobramycin and Vancomycin Exposure Required to Eradicate Biofilms on Muscle and Bone Tissue In Vitro, J. Bone Jt. Infect., 4, 1-9, https://doi.org/10.7150/jbji.29711, 2019.

Bassetti, M., Vitale, F., Melica, G., Righi, E., Di Biagio, A., Molfetta, L., Pipino, F., Cruciani, M., and Bassetti, D.: Linezolid in the treatment of Gram-positive prosthetic joint infections, J. Antimicrob. Chemother., 55, 387-390, https://doi.org/10.1093/jac/dki016, 2005.

Calabro, F., Coen, M., Franceschini, M., Franco-Cendejas, R., Hewlett, A., Segreti, J., and Senneville, E.: Hip and Knee Section, Treatment, Antimicrobial Suppression: Proceedings of International Consensus on Orthopedic Infections, J. Arthroplasty, 34, S483-S485, https://doi.org/10.1016/j.arth.2018.09.034, 2019.

Cobo, J., Lora-Tamayo, J., Euba, G., Jover-Saenz, A., Palomino, J., del Toro, M. D., Rodriguez-Pardo, D., Riera, M., Ariza, J., and Red Espanola para la Investigacion en Patologia, I.: Linezolid in late-chronic prosthetic joint infection caused by grampositive bacteria, Diagn. Microbiol. Infect. Dis., 76, 93-98, https://doi.org/10.1016/j.diagmicrobio.2013.02.019, 2013.

Corona, P. S., Espinal, L., Rodriguez-Pardo, D., Pigrau, C., Larrosa, N., and Flores, X.: Antibiotic susceptibility in grampositive chronic joint arthroplasty infections: increased aminoglycoside resistance rate in patients with prior aminoglycosideimpregnated cement spacer use, J. Arthroplasty, 29, 1617-1621, https://doi.org/10.1016/j.arth.2014.03.029, 2014.

de Beaubien, B., Belden, K., Bell, K., Boyle, K., Cordero-Ampuero, J., Della Valle, C. J., Eijer, H., Ferry, C., Janz, V., Kessler, B., Kratky, A., Lachiewicz, A., Martin, K. E., Murillo, O., Nijhof, M., Nodzo, S. R., O'Callaghan, M., Petrie, M. J., Stockley, I., and Suleiman, L. I.: Hip and Knee Section, Treatment, Antimicrobials: Proceedings of International Consensus on Orthopedic Infections, J. Arthroplasty, 34, S477-S482, https://doi.org/10.1016/j.arth.2018.09.033, 2019.

Deroche, L., Plouzeau, C., Bemer, P., Tande, D., Valentin, A. S., Jolivet-Gougeon, A., Lemarie, C., Bret, L., Kempf, M., Hery-Arnaud, G., Corvec, S., Burucoa, C., Arvieux, C., Bernard, L., and and the, C. S. G.: Probabilistic chemotherapy in knee and hip replacement infection: the place of linezolid, Eur. J. Clin. Microbiol. Infect. Dis., 38, 1659-1663, https://doi.org/10.1007/s10096-019-03594-z, 2019.

De Vecchi, E., George, D. A., Romano, C. L., Pregliasco, F. E., Mattina, R., and Drago, L.: Antibiotic sensitivities of coagulasenegative staphylococci and Staphylococcus aureus in hip and knee periprosthetic joint infections: does this differ if patients meet the International Consensus Meeting Criteria?, Infect. Drug Resist., 11, 539-546, https://doi.org/10.2147/IDR.S151271, 2018.

Drago, L., De Vecchi, E., Bortolin, M., Zagra, L., Romano, C. L., and Cappelletti, L.: Epidemiology and Antibiotic Resistance of Late Prosthetic Knee and Hip Infections, J. Arthroplasty, 32, 2496-2500, https://doi.org/10.1016/j.arth.2017.03.005, 2017. 
Edelstein, A. I., Okroj, K. T., Rogers, T., Della Valle, C. J., and Sporer, S. M.: Systemic Absorption of Antibiotics From Antibiotic-Loaded Cement Spacers for the Treatment of Periprosthetic Joint Infection, J. Arthroplasty, 33, 835-839, https://doi.org/10.1016/j.arth.2017.09.043, 2018.

Eriksson, H. K., Ahadpour, D., Hailer, N. P., Lazarinis, S., and Jarhult, J. D.: Linezolid in the treatment of periprosthetic joint infection caused by coagulasenegative staphylococci, Infect. Dis. (Lond.), 51, 683-690, https://doi.org/10.1080/23744235.2019.1642510, 2019.

Gandelman, K., Zhu, T., Fahmi, O. A., Glue, P., Lian, K., Obach, R. S., and Damle, B.: Unexpected effect of rifampin on the pharmacokinetics of linezolid: in silico and in vitro approaches to explain its mechanism, J. Clin. Pharmacol., 51, 229-236, https://doi.org/10.1177/0091270010366445, 2011.

George, J., Newman, J. M., Klika, A. K., Miller, E. M., Tan, T. L., Parvizi, J., and Higuera, C. A.: Changes in Antibiotic Susceptibility of Staphylococcus aureus Between the Stages of 2Stage Revision Arthroplasty, J. Arthroplasty, 33, 1844-1849, https://doi.org/10.1016/j.arth.2018.01.056, 2018.

Gomez, J., Canovas, E., Banos, V., Martinez, L., Garcia, E., Hernandez-Torres, A., Canteras, M., Ruiz, J., Medina, M., Martinez, P., Canovas, A., Soriano, A., and Clavel, M.: Linezolid plus rifampin as a salvage therapy in prosthetic joint infections treated without removing the implant, Antimicrob. Agents Chemother., 55, 4308-4310, https://doi.org/10.1128/AAC.00352-11, 2011.

Harwood, P. J., Talbot, C., Dimoutsos, M., Sunderland, G., Shaw, D., Wilcox, M. H., and Giannoudis, P. V.: Early experience with linezolid for infections in orthopaedics, Injury, 37, 818-826, https://doi.org/10.1016/j.injury.2006.02.007, 2006.

Hipfl, C., Winkler, T., Janz, V., Perka, C., and Muller, M.: Management of Chronically Infected Total Knee Arthroplasty With Severe Bone Loss Using Static Spacers With Intramedullary Rods, J. Arthroplasty, 34, 1462-1469, https://doi.org/10.1016/j.arth.2019.03.053, 2019.

Joel, J., Graham, S. M., Peckham-Cooper, A., Korres, N., Tsouchnica, H., and Tsiridis, E.: Clinical results of linezolid in arthroplasty and trauma MRSA related infections, World J. Orthop., 5, 151-157, https://doi.org/10.5312/wjo.v5.i2.151, 2014.

Khan, N., Parmar, D., Ibrahim, M. S., Kayani, B., and Haddad, F. S.: Outcomes of repeat two-stage exchange hip arthroplasty for prosthetic joint infection, Bone Joint J., 101-B, 110-115, https://doi.org/10.1302/0301-620X.101B6.BJJ-20181556.R1, 2019.

Kheir, M. M., Tan, T. L., Gomez, M. M., Chen, A. F., and Parvizi, J.: Patients With Failed Prior Two-Stage Exchange Have Poor Outcomes After Further Surgical Intervention, J. Arthroplasty, 32, 1262-1265, https://doi.org/10.1016/j.arth.2016.10.008, 2017.

Kutscha-Lissberg, F., Hebler, U., Muhr, G., and Koller, M.: Linezolid penetration into bone and joint tissues infected with methicillin-resistant staphylococci, Antimicrob. Agents Chemother., 47, 3964-3966, https://doi.org/10.1128/aac.47.12.3964-3966.2003, 2003.

Legout, L., Valette, M., Dezeque, H., Nguyen, S., Lemaire, X., Loiez, C., Caillaux, M., Beltrand, E., Dubreuil, L., Yazdanpanah, Y., Migaud, H., and Senneville, E.: Tolerability of prolonged linezolid therapy in bone and joint infection: protective effect of rifampicin on the occurrence of anaemia?, J. Antimicrob.
Chemother., 65, 2224-2230, https://doi.org/10.1093/jac/dkq281, 2010.

Leijtens, B., Weerwag, L., Schreurs, B. W., Kullberg, B. J., and Rijnen, W.: Clinical Outcome of Antibiotic Suppressive Therapy in Patients with a Prosthetic Joint Infection after Hip Replacement, J. Bone Jt. Infect., 4, 268-276, https://doi.org/10.7150/jbji.37262, 2019.

Lourtet-Hascoet, J., Felice, M. P., Bicart-See, A., Bouige, A., Giordano, G., and Bonnet, E.: Species and antimicrobial susceptibility testing of coagulase-negative staphylococci in periprosthetic joint infections, Epidemiol. Infect., 146, 1771-1776, https://doi.org/10.1017/S0950268818001437, 2018.

Lu, P. L., Wang, J. T., Chen, C. J., Chen, W. C., Chen, T. C., Hwang, Y. C., and Chang, S. C.: Compassionate use of linezolid for adult Taiwanese patients with bone and joint infections, Chemotherapy, 56, 429-435, https://doi.org/10.1159/000317752, 2010.

Metsemakers, W. J., Kuehl, R., Moriarty, T. F., Richards, R. G., Verhofstad, M. H. J., Borens, O., Kates, S., and Morgenstern, M.: Infection after fracture fixation: Current surgical and microbiological concepts, Injury, 49, 511-522, https://doi.org/10.1016/j.injury.2016.09.019, 2018.

Moher, D., Liberati, A., Tetzlaff, J., Altman, D. G., and Group, P.: Preferred reporting items for systematic reviews and metaanalyses: the PRISMA statement, PLoS Med., 6, e1000097, https://doi.org/10.1371/journal.pmed.1000097, 2009.

Morata, L., Senneville, E., Bernard, L., Nguyen, S., Buzele, R., Druon, J., Tornero, E., Mensa, J., and Soriano, A.: A Retrospective Review of the Clinical Experience of Linezolid with or Without Rifampicin in Prosthetic Joint Infections Treated with Debridement and Implant Retention, Infect. Dis. Ther., 3, 235-243, https://doi.org/10.1007/s40121-014-0032-z, 2014a.

Morata, L., Tornero, E., Martinez-Pastor, J. C., Garcia-Ramiro, S., Mensa, J., and Soriano, A.: Clinical experience with linezolid for the treatment of orthopaedic implant infections, J. Antimicrob. Chemother., 69 (Suppl. 1), i47-52, https://doi.org/10.1093/jac/dku252, 2014b.

Moriarty, T. F., Kuehl, R., Coenye, T., Metsemakers, W. J., Morgenstern, M., Schwarz, E. M., Riool, M., Zaat, S. A. J., Khana, N., Kates, S. L., and Richards, R. G.: Orthopaedic device-related infection: current and future interventions for improved prevention and treatment, EFORT Open Rev., 1, 89-99, https://doi.org/10.1302/2058-5241.1.000037, 2016.

Nguyen, S., Pasquet, A., Legout, L., Beltrand, E., Dubreuil, L., Migaud, H., Yazdanpanah, Y., and Senneville, E.: Efficacy and tolerance of rifampicin-linezolid compared with rifampicincotrimoxazole combinations in prolonged oral therapy for bone and joint infections, Clin. Microbiol. Infect., 15, 1163-1169, https://doi.org/10.1111/j.1469-0691.2009.02761.x, 2009.

OCEBM Levels of Evidence Working Group (Jeremy Howick, Iain Chalmers, Paul Glasziou, Trish Greenhalgh, Carl Heneghan, Alessandro Liberati, Ivan Moschetti, Bob Phillips, Hazel Thornton, and Olive Goddard and Mary Hodgkinson): The Oxford Levels of Evidence 2", Oxford Centre for Evidence-Based Medicine, 2011.

Osmon, D. R., Berbari, E. F., Berendt, A. R., Lew, D., Zimmerli, W., Steckelberg, J. M., Rao, N., Hanssen, A., Wilson, W. R., and Infectious Diseases Society of America: Executive summary: diagnosis and management of prosthetic joint infection: clinical practice guidelines by the Infectious Diseases Society 
of America, Clinical infectious diseases: an official publication of the Infectious Diseases Society of America, 56, 1-10, https://doi.org/10.1093/cid/cis966, 2013a.

Osmon, D. R., Berbari, E. F., Berendt, A. R., Lew, D., Zimmerli, W., Steckelberg, J. M., Rao, N., Hanssen, A., Wilson, W. R., and Infectious Diseases Society of America: Diagnosis and management of prosthetic joint infection: clinical practice guidelines by the Infectious Diseases Society of America, Clin. Infect. Dis., 56, e1-e25, https://doi.org/10.1093/cid/cis803, 2013b.

Oussedik, S. I. and Haddad, F. S.: The use of linezolid in the treatment of infected total joint arthroplasty, J. Arthroplasty, 23, 273278, https://doi.org/10.1016/j.arth.2007.03.022, 2008.

Papadopoulos, A., Plachouras, D., Giannitsioti, E., Poulakou, G., Giamarellou, H., and Kanellakopoulou, K.: Efficacy and tolerability of linezolid in chronic osteomyelitis and prosthetic joint infections: a case-control study, J. Chemother., 21, 165-169, https://doi.org/10.1179/joc.2009.21.2.165, 2009.

Parvizi, J., Tan, T. L., Goswami, K., Higuera, C., Della Valle, C., Chen, A. F., and Shohat, N.: The 2018 Definition of Periprosthetic Hip and Knee Infection: An EvidenceBased and Validated Criteria, J. Arthroplasty, 33, 1309-1314, https://doi.org/10.1016/j.arth.2018.02.078, 2018.

Pea, F., Furlanut, M., Cojutti, P., Cristini, F., Zamparini, E., Franceschi, L., and Viale, P.: Therapeutic drug monitoring of linezolid: a retrospective monocentric analysis, Antimicrob. Agents Chemother., 54, 4605-4610, https://doi.org/10.1128/AAC.00177-10, 2010.

Pea, F., Viale, P., Cojutti, P., Del Pin, B., Zamparini, E., and Furlanut, M.: Therapeutic drug monitoring may improve safety outcomes of long-term treatment with linezolid in adult patients, J. Antimicrob. Chemother., 67, 2034-2042, https://doi.org/10.1093/jac/dks153, 2012.

Rao, N. and Hamilton, C. W.: Efficacy and safety of linezolid for Gram-positive orthopedic infections: a prospective case series, Diagn. Microbiol. Infect. Dis., 59, 173-179, https://doi.org/10.1016/j.diagmicrobio.2007.04.006, 2007.

Razonable, R. R., Osmon, D. R., and Steckelberg, J. M.: Linezolid therapy for orthopedic infections, Mayo. Clin. Proc., 79, 11371144, https://doi.org/10.4065/79.9.1137, 2004.

Sendi, P. and Zimmerli, W.: Antimicrobial treatment concepts for orthopaedic device-related infection, Clin. Microbiol. Infect., 18, 1176-1184, https://doi.org/10.1111/1469-0691.12003, 2012.

Signore, A., Sconfienza, L. M., Borens, O., Glaudemans, A., Cassar-Pullicino, V., Trampuz, A., Winkler, H., Gheysens, O., Vanhoenacker, F., Petrosillo, N., and Jutte, P. C.: Consensus document for the diagnosis of prosthetic joint infections: a joint paper by the EANM, EBJIS, and ESR (with ESCMID endorsement), Eur. J. Nucl. Med. Mol. Imaging, 46, 971-988, https://doi.org/10.1007/s00259-019-4263-9, 2019.

Siqueira, M. B., Saleh, A., Klika, A. K., O’Rourke, C., Schmitt, S., Higuera, C. A., and Barsoum, W. K.: Chronic Suppression of Periprosthetic Joint Infections with Oral Antibiotics Increases Infection-Free Survivorship, J. Bone Joint Surg. Am., 97, 12201232, https://doi.org/10.2106/JBJS.N.00999, 2015.

Slim, K., Nini, E., Forestier, D., Kwiatkowski, F., Panis, Y., and Chipponi, J.: Methodological index for non-randomized studies (minors): development and validation of a new instrument, ANZ J. Surg., 73, 712-716, https://doi.org/10.1046/j.14452197.2003.02748.x, 2003.
Soriano, A., Gomez, J., Gomez, L., Azanza, J. R., Perez, R., Romero, F., Pons, M., Bella, F., Velasco, M., and Mensa, J.: Efficacy and tolerability of prolonged linezolid therapy in the treatment of orthopedic implant infections, Eur. J. Clin. Microbiol. Infect. Dis., 26, 353-356, https://doi.org/10.1007/s10096007-0289-1, 2007.

Takoudju, E., Bemer, P., Touchais, S., Asseray, N., Corvec, S., Khatchatourian, L., Serandour, N., Boutoille, D., Nantes Bone and Joint Infections Study Group: Bacteriological relevance of linezolid vs. vancomycin in postoperative empirical treatment of osteoarticular infections: a retrospective single-center study, Int. J. Antimicrob. Agents, 52, 663-666, https://doi.org/10.1016/j.ijantimicag.2018.04.022, 2018.

Tattevin, P., Arvieux, C., and Michelet, C.: What is the place of teicoplanin and linezolid in the treatment of prosthetic joint infections?, Clin. Microbiol. Infect., 12, 1241-1242, https://doi.org/10.1111/j.1469-0691.2006.01560.x, 2006.

Tevell, S., Baig, S., Nilsdotter-Augustinsson, A., Stegger, M., and Soderquist, B.: Same Organism, Different Phenotype - Are Phenotypic Criteria Adequate In Coagulase-Negative Staphylococcal Orthopaedic Implant-Associated Infections?, J. Bone Jt. Infect., 4, 16-19, https://doi.org/10.7150/jbji.30256, 2019.

Thompson, J. M., Saini, V., Ashbaugh, A. G., Miller, R. J., Ordonez, A. A., Ortines, R. V., Wang, Y., Sterling, R. S., Jain, S. K., and Miller, L. S.: Oral-Only Linezolid-Rifampin Is Highly Effective Compared with Other Antibiotics for Periprosthetic Joint Infection: Study of a Mouse Model, J. Bone Joint Surg. Am., 99, 656665, https://doi.org/10.2106/JBJS.16.01002, 2017.

Tornero, E., Morata, L., Martinez-Pastor, J. C., Angulo, S., Combalia, A., Bori, G., Garcia-Ramiro, S., Bosch, J., Mensa, J., and Soriano, A.: Importance of selection and duration of antibiotic regimen in prosthetic joint infections treated with debridement and implant retention, J. Antimicrob. Chemother., 71, 13951401, https://doi.org/10.1093/jac/dkv481, 2016.

Vazquez, J. A., Arnold, A. C., Swanson, R. N., Biswas, P., and Bassetti, M.: Safety of long-term use of linezolid: results of an open-label study, Ther. Clin. Risk Manag., 12, 1347-1354, https://doi.org/10.2147/TCRM.S109444, 2016.

Winkler, T., Stuhlert, M. G. W., Lieb, E., Muller, M., von Roth, P., Preininger, B., Trampuz, A., and Perka, C. F.: Outcome of short versus long interval in two-stage exchange for periprosthetic joint infection: a prospective cohort study, Arch. Orthop. Trauma Surg., 139, 295-303, https://doi.org/10.1007/s00402-018-30524, 2019.

Wouthuyzen-Bakker, M., Nijman, J. M., Kampinga, G. A., van Assen, S., and Jutte, P. C.: Efficacy of Antibiotic Suppressive Therapy in Patients with a Prosthetic Joint Infection, J. Bone Jt. Infect., 2, 77-83, https://doi.org/10.7150/jbji.17353, 2017.

Zimmerli, W. and Sendi, P.: Role of Rifampin against Staphylococcal Biofilm Infections In Vitro, in Animal Models, and in Orthopedic-Device-Related Infections, Antimicrob. Agents Chemother., 63, e01746-18, https://doi.org/10.1128/AAC.01746-18, 2019. 